\title{
Evaluation, Diagnosis and Management of Popliteal Artery Aneurysms: A Clinical Review
}

\author{
Fernando N Aguila ${ }^{\mathrm{a}, \mathrm{b}}$, Thomas H. Hartranft ${ }^{\mathrm{a}}$, Ghaleb A. Hannun", \\ Charles A. Hartranft ${ }^{\mathrm{a}}$, Charles R. Holden ${ }^{\mathrm{a}}$
}

\begin{abstract}
Aneurysmal disease of the popliteal artery is more common than historically thought with most of the cases going unrecognized, many times until ischemic symptoms to the leg brings it to the physician's attention. The fact that the popliteal artery is the most common location for peripheral artery aneurysms (PAA), and it is easily accessible to physical examination, makes necessary not only for surgeons, but all physicians to have a basic knowledge of this entity. The common pathophysiological defect where most of the etiologies converge seems to be degeneration of the artery media through a variety of mechanisms including metalloproteinases. Since the most important etiologic factor is atherosclerosis, it is expected that these patients will have other vascular manifestations in multiple other locations. Once clearly diagnosed, PAA can be treated with surgical ligation or resection of the aneurysmal sac and bypass. As with other vascular surgery problems, endovascular techniques have evolved to become more reliable as methods of treatment with growing technical success rates and patency rates.
\end{abstract}

Keywords: Popliteal; Artery; Aneurysm; Endovascular; Peripheral

\section{Introduction}

An aneurysm is defined as a permanent and irreversible localized dilation of a blood vessel to at least a $50 \%$ increase in diameter compared with the expected normal (Fig. 1). Popliteal artery aneurysms (PAA) were first described by John Hunter more than four centuries ago. They are the most common peripheral artery aneurysms ( $67 \%$ of all), with a preva-

Manuscript accepted for publication May 13, 2013

\footnotetext{
${ }^{a}$ Department of surgery, Mount Carmel Health System, 793 West State Street, Columbus Ohio, 43222-1551, USA

${ }^{\mathrm{b}}$ Corresponding author: Fernando N Aguila, 793 West State street, Columbus Ohio, 43222-1551, USA. Email: faguila@mchs.com
}

doi: http://dx.doi.org/10.4021/jcs174w lence of nearly $1 \%$ in elderly males [1]. This is likely an underestimation; however, since many patients are asymptomatic. PAA incidence is increasing likely due to improved imaging technology and a growing elderly population. This disease is more common in males by 30 -fold [2]. The most commonly present incidentally as a pulsatile mass behind the knee on physical exam. Those diagnosed are often patients with significant cardiovascular risk factors and symptoms of chronic occlusive arterial disease. Synchronous aneurysms in other locations are common with about $50 \%$ of patients having a contralateral PAA, $40-70 \%$ an abdominal aortic aneurysm (AAA), and $40 \%$ a femoral artery aneurysm simultaneously [1]. Certainly, the most dramatic presentation is that of acute ischemic symptoms from thrombosis, embolism or both. Some patients will present with complains of a

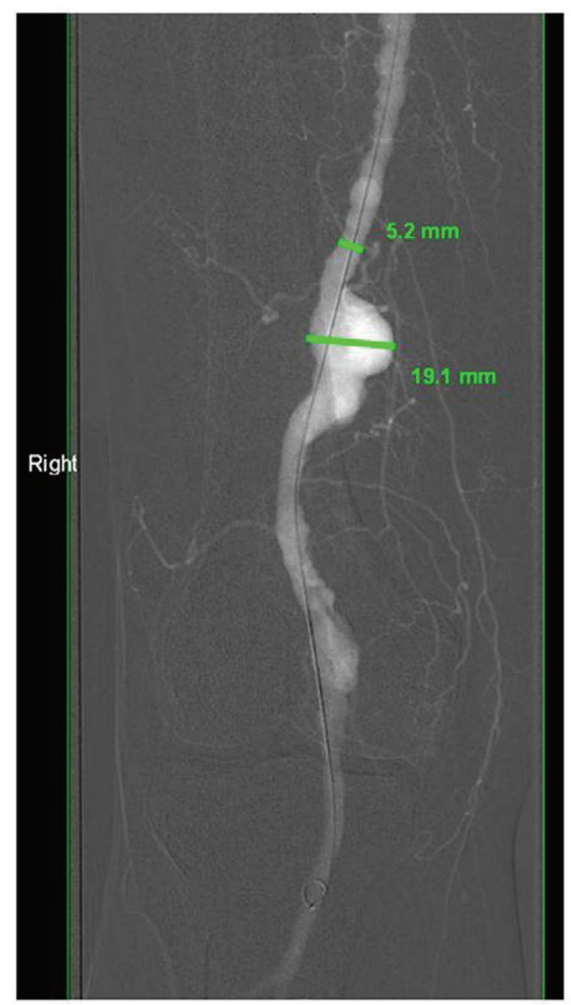

Figure 1. Angiogram showing right politeal aneurysm. 

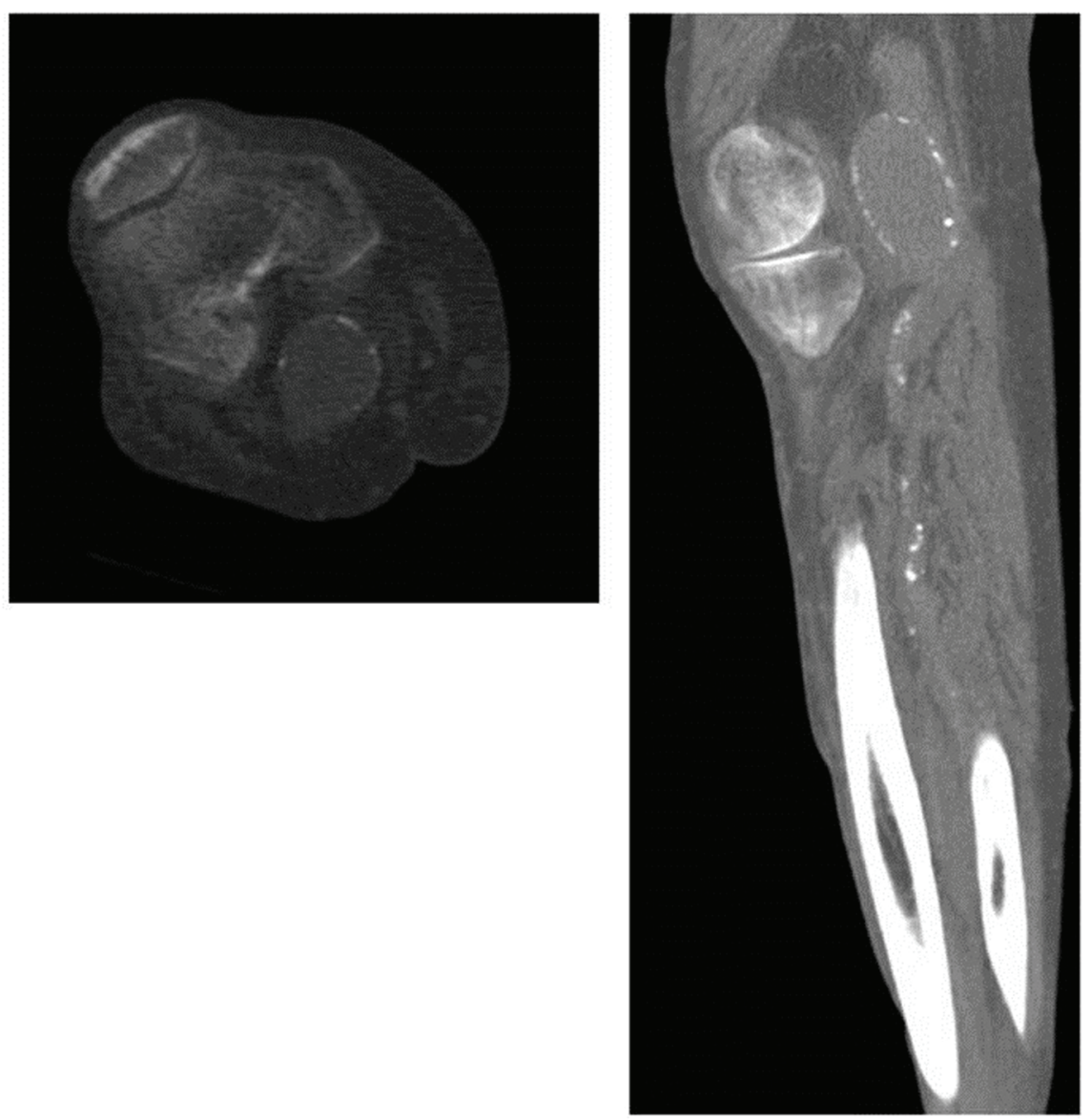

Figure 2. Representative images of CT of the leg with intravenous contrast showing a politeal aneurysm in the transverse (above) and coronal (right) views.

pulsatile mass behind the knee.

\section{Etiology and Pathophysiology}

The etiologic factors associated with PAA are most commonly atherosclerosis and post-obstructive angioectasia. Infectious processes are less frequent but remain a well documented etiology factor. Regardless of the etiology, common pathophysiologic factors found include media and adventitial degeneration by metalloproteinases and depletion of smooth muscle cells. The fact that some PAA develop distal to stenotic areas (post-obstructive aneurysm) suggests turbulent flow as a possible contributing factor. Other associated factors include cigarette smoking, hypertension and cardiac disease. Diabetes is more common in patients with PAA than the general population but is actually associated with slower progression rates compared to non diabetic patients. Multiple pathophysiological paths eventually confluence to weakening the media layer or the vessel through degradation of elastin and collagen, matrix metalloproteinases and oxygen radicals play a central role on this. Bechet's and Marfan's syndromes are associated with an increased incidence of PAA but there are likely multiple genetic factors that have yet to be elucidated [1].

\section{Natural History and Clinical Presentation}

The natural history of PAA is less well defined than that of AAA. The progression rate has been estimated to be 0.7 to $1.5 \mathrm{~mm}$ per year for PAA smaller than $2 \mathrm{~cm}$ and 1.5 to 3.7 $\mathrm{mm} /$ year for those larger than $2 \mathrm{~cm}$. Size alone however, does not necessarily affect outcomes for PAA compared to aneurysms in other locations. This is likely due to the low risk of rupture but yet a significant thrombo-embolic risk that seems to have limited correlation to size $[3,4]$.

A significant proportion of patients with PAA are asymptomatic, but the complication rate increases over time with 18 to $33 \%$ of PAA eventually developing symptoms of thrombo-embolism or mass effect. Every year $5-24 \%$ patients develop symptoms while being observed $[1,5,6]$. 


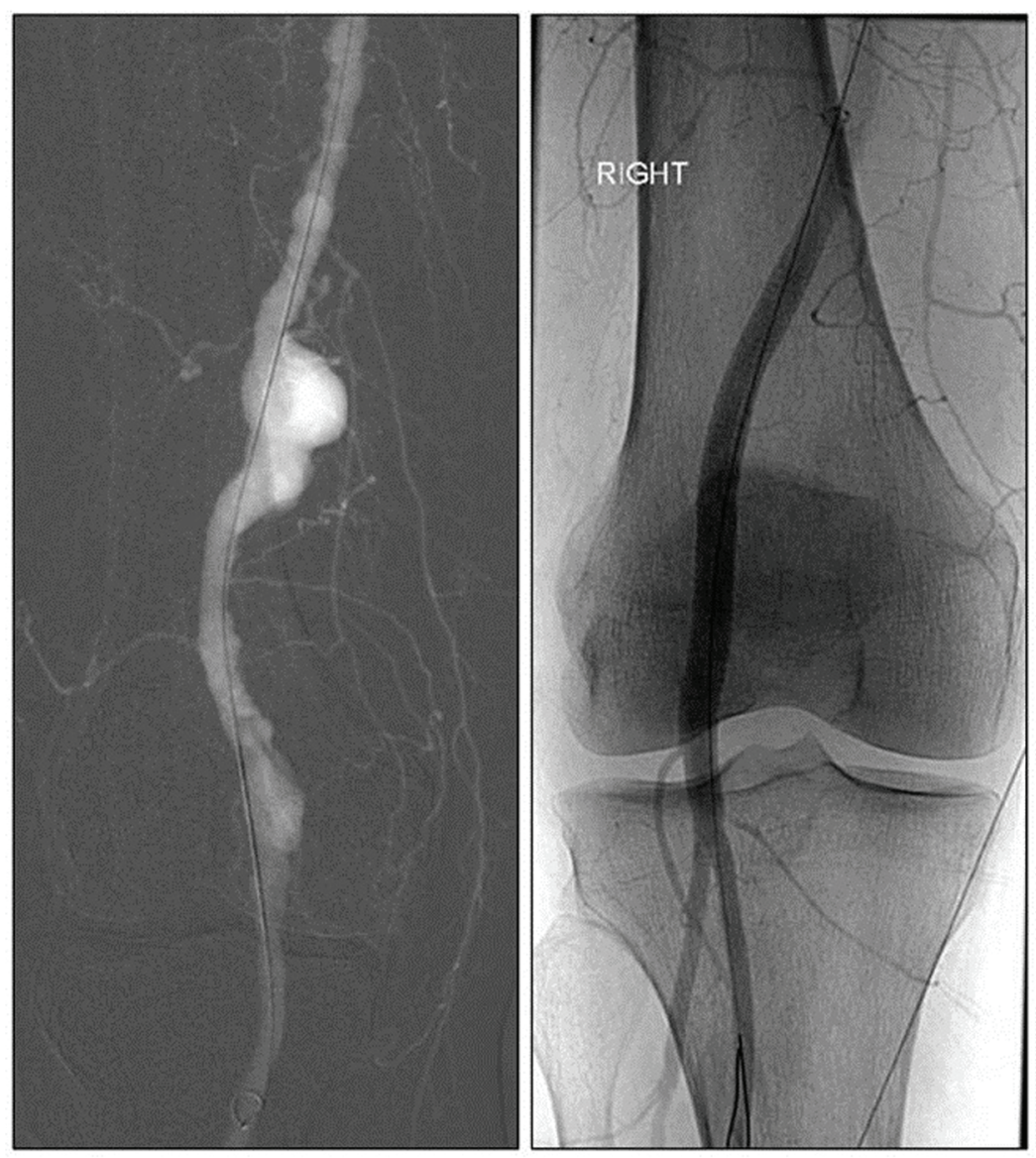

Figure 3. Angiographic image of a politeal aneurysm before (left) and after (right) endovascular exclusion with a flexible stent.

Of the asymptomatic patients followed clinically and with imaging, ischemia will eventually occur in one-third, intermittent claudication in $25 \%$ and popliteal vein compression in $5 \%$.

In addition to size, minor trauma, tortuosity and distortion are other risk factors for thrombosis. Depending on the acuity of the thrombosis the extremity may be at risk of irreversible ischemia if poor collateral circulation has developed to bypass the obstruction.

Interestingly, several reports suggest a higher rate of thrombosis in smaller aneurysms compared to large ones [4]. It is unknown if this is an observational bias or real phenomenon with still an unknown pathophysiological explanation.

The size of the aneurysm is directly proportional to the risk of rupture. In a series of 116 popliteal aneurysms, the median diameter of asymptomatic disease was $2.0 \mathrm{~cm} ; 3.0$ $\mathrm{cm}$ was the average size of those producing ischemia; and those producing compressive symptoms were $3.45 \mathrm{~cm}$ in average. Compared to AAA, the incidence of rupture is traditionally recognized as quite low, with reports ranging from $0.1-2.8 \%$ even for larger aneurysms [7]. PAA appears to be a progressive disease process encompassing a continuum of symptom free disease, minimally/chronically symptomatic disease, and ultimately limb threatening ischemia.

\section{Screening and Management}

Currently the best screening method for PAA in the general population is still a careful history and physical exam. Screening imaging is not a cost effective epidemiological intervention for the general population, but it is still unknown what specific high risk groups would benefit from screening in the primary care physician's office. Duplex ultrasound provides adequate visualization for diagnosis and to determine patency but computer tomography and angiogram can show detail necessary for preoperative planning (Fig. 2). Once an aneurysm is found clinically or highly suspected, the diagnostic workup should include the contralateral extremity and the abdominal and pelvic vasculature in all cases due to the high risk of synchronous aneurysmal disease [8].

Traditional recommendations of repairing asymptomatic PAA that are $2 \mathrm{~cm}$ or larger in diameter, symptomatic, or thrombosed are still valid $[4,9]$. Asymptomatic popliteal 
aneurysms with minimal or no tortuosity are best managed with observation via arterial Duplex exams every $6-12$ months. This especially holds true for older individuals or those with significant co-morbidities. Despite the fact previous reports comparing repair of minimally symptomatic PAA's to asymptomatic disease did not show significantly different outcomes, elective repair anecdotally carries a better overall prognosis. This appears evident when comparing limb loss rates of $<1 \%$ in minimally symptomatic to asymptomatic patients versus $16-43 \%$ if severe ischemia is present [3].

Aneurysm exclusion and autologous vein bypass graft is the surgical therapy of choice. PTFE prosthesis is a suboptimal but feasible alternative if no vein is available for reconstruction. The standard open approach is performed in one of two ways. A medial approach involves ligation or partial excision of the diseased arterial segment and subsequent above-knee to below-knee bypass. In contrast, the posterior approach often utilizes resection of the aneurysm with reconstruction via interposition grafting. While both techniques yield similar patency and complication rates, there have been increasing reports of persistent perfusion of the aneurismal sac with the medial approach [10]. Patency rates following open repair have been reported at $70-90 \%$ for 1 - 2 years [11]. Complications associated with surgical repair of PAA include DVT, lymph leakage, graft infection, cardiac problems, and others [6]. After elective repair, the 5 year limb salvage and graft patency rates are 90 and $80 \%$ respectively with $1 \%$ patients having residual symptoms $[1$, 2].

As is other fields, minimally invasive approaches have become an additional tool for treatment of PAA (Fig. 3). Endovascular stenting recently evolved as a feasible and safe alternative with patency rates similar to open repair as published by several groups [12-17]. Although 2-year primary and secondary patency rates have being reported to be $60-85 \%$ and $80-100 \%$ respectively, no randomized controlled trials have been reported to compare endovascular with open approaches. The largest series of PAA treated with endovascular repair is by Tielliu et al. where the reported primary and secondary patency rates were $70 \%$ and $76 \%$ at 5 years respectively. Clopidogrel administration and surgeon experience were both noted to be statistically significant as favorable prognostic factors [18]. If aneurysmal thrombosis is present at the time of repair, thrombolysis therapy to runoff vessels to assure distal patency has been recommended by many authors. There are no absolute contraindications to the endovascular approach but poor distal run-off vessels predicts poor prognosis after endovascular repair even when thrombolysis is part of the treatment plan $[15,16]$. Patients with large aneurysms and mass effect, such as venous stasis and nerve compression, are not suitable candidates for endovascular repair for the same reasons than aneurysm exclusion and bypass would not be a good option, these pro-

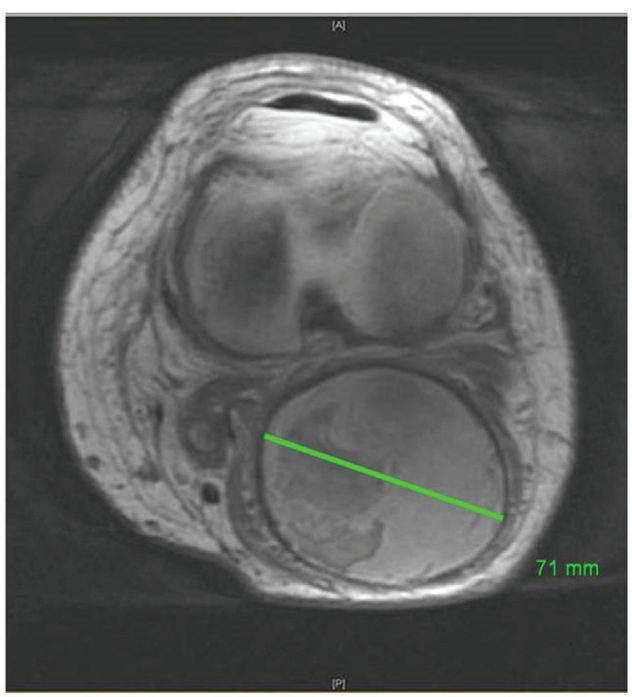

Figure 4. Representative image of MRI of right knee shows a surgically excluded $7 \mathrm{~cm}$ politeal aneurysm causing pain and mass effect.

cedures would not resolve the mass effect of the PAA itself (Fig. 4). A possible exception to this is the unstable elderly individual with a thrombosed PAA as a temporizing measure before definitive open excision of the compressing aneurysm [3]. Recent reports have emerged exploring endovascular intervention for PAA repair in both elective and emergency situations. Trinidad-Hernandez et al. reported a 97\% technical success rate for a study group undergoing both elective and emergent repair. The primary patency at 1 -year was $86 \%$ (elective; $95 \%$, emergent; $69 \%$ ) while secondary patency for the same time period was $91 \%$ (elective; $100 \%$, emergent; $91 \%$ ). While elective and emergent endovascular repair remains a feasible treatment modality for anatomically suitable patients, adverse events such as stent thrombosis occur rather frequently and more so in the setting of emergent repairs [19].

\section{Conclusions}

Popliteal aneurysmal disease may be more prevalent than previously estimated. It represents a potential and serious threat not only to the patient's limb function and viability but also his/her life. It is associated with traditional cardiovascular risk factors and frequently co-exists with aneurismal disease in other locations. Open ligation and bypass with greater saphenous vein is the gold standard for treatment, but novel endovascular techniques have come to play a major role in the armamentarium for intervention in both the emergency and elective scenarios. As endovascular technologies continue to evolve and improve, endovascular repair will play a larger role in intervention strategies across the coun- 
try. There remains however, a greater need for further high volume and prospective data to solidify its utility and safety.

\section{Conflict of Interest}

None declared.

\section{References}

1. Galland RB. Popliteal aneurysms: from John Hunter to the 21st century. Ann R Coll Surg Engl. 2007;89(5):466471.

2. Michaels JA, Galland RB. Management of asymptomatic popliteal aneurysms: the use of a Markov decision tree to determine the criteria for a conservative approach. Eur J Vasc Surg. 1993;7(2):136-143.

3. Goncu T, Tiryakioglu O, Sezen M, Yavuz S. Giant popliteal aneurysm with deep vein thrombosis, foot drop and arteriomegali. BMJ Case Rep. 2009;2009

4. Miani S, Boneschi M, Giuffrida GF, Beretta L, Giordanengo F. [Aneurysm of the popliteal artery. Prognostic and therapeutic criteria]. Minerva Cardioangiol. 1993;41(7-8):319-323.

5. Gifford RW, Jr., Hines EA, Jr., Janes JM. An analysis and follow-up study of one hundred popliteal aneurysms. Surgery. 1953;33(2):284-293.

6. Dawson I, van Bockel JH, Brand R, Terpstra JL. Popliteal artery aneurysms. Long-term follow-up of aneurysmal disease and results of surgical treatment. J Vasc Surg. 1991;13(3):398-407.

7. Taurino M, Calisti A, Grossi R, Maggiore C, Speziale F, Fiorani P. Outcome after early treatment of popliteal artery aneurysms. Int Angiol. 1998;17(1):28-33.

8. Agrafiotis AC, Horn D, Segers B, Lemaitre J, Bosschaerts T. Ruptured aneurysm of the popliteal artery. Is the diagnosis still difficult? Minerva Chir. 2012;67(4):355-360.

9. Szilagyi DE, Schwartz RL, Reddy DJ. Popliteal arterial aneurysms. Their natural history and management. Arch Surg. 1981;116(5):724-728.

10. Tsilimparis N, Dayama A, Ricotta JJ, 2nd. Open and en- dovascular repair of popliteal artery aneurysms: tabular review of the literature. Ann Vasc Surg. 2013;27(2):259265.

11. Ascer E, Collier P, Gupta SK, Veith FJ. Reoperation for polytetrafluoroethylene bypass failure: the importance of distal outflow site and operative technique in determining outcome. J Vasc Surg. 1987;5(2):298-310.

12. Rajasinghe HA, Tzilinis A, Keller T, Schafer J, Urrea $\mathrm{S}$. Endovascular exclusion of popliteal artery aneurysms with expanded polytetrafluoroethylene stent-grafts: early results. Vasc Endovascular Surg. 2006;40(6):460-466.

13. Ihlberg LH, Roth WD, Alback NA, Kantonen IK, Lepantalo M. Successful percutaneous endovascular treatment of a ruptured popliteal artery aneurysm. J Vasc Surg. 2000;31(4):794-797.

14. Antonello M, Frigatti P, Battocchio P, Lepidi S, Cognolato D, Dall'Antonia A, Stramana R, et al. Open repair versus endovascular treatment for asymptomatic popliteal artery aneurysm: results of a prospective randomized study. J Vasc Surg. 2005;42(2):185-193.

15. Garg K, Rockman CB, Kim BJ, Jacobowitz GR, Maldonado TS, Adelman MA, Veith FJ, et al. Outcome of endovascular repair of popliteal artery aneurysm using the Viabahn endoprosthesis. J Vasc Surg. 2012;55(6):16471653.

16. Pulli R, Dorigo W, Fargion A, Pratesi G, Innocenti AA, Angiletta D, Pratesi C. Comparison of early and midterm results of open and endovascular treatment of popliteal artery aneurysms. Ann Vasc Surg. 2012;26(6):809-818.

17. Jung E, Jim J, Rubin BG, Sanchez LA, Choi ET, Sicard GA, Geraghty PJ. Long-term outcome of endovascular popliteal artery aneurysm repair. Ann Vasc Surg. 2010;24(7):871-875.

18. Tielliu IF, Verhoeven EL, Zeebregts CJ, Prins TR, Span MM, van den Dungen JJ. Endovascular treatment of popliteal artery aneurysms: results of a prospective cohort study. J Vasc Surg. 2005;41(4):561-567.

19. Trinidad-Hernandez M, Ricotta JJ, 2nd, Gloviczki P, Kalra M, Oderich GS, Duncan AA, Bower TC. Results of elective and emergency endovascular repairs of popliteal artery aneurysms. J Vasc Surg. 2013;57(5):12991305 . 